\title{
TRAVEL VACCINATION HEADACHES
}

T he rising number of people travelling overseas in the past 20 years has led to a higher risk of exposure to diseases not commonly found in Australia. It has also led to a demand for country-specific travel health advice which has not always been found easily. The Western Sydney Public Health Unit has been responsible for dealing with public and professional inquiries about travel health since its inception. Demand on time and resources to satisfy this need has risen dramatically.

Between October 11 and December 14, 1990, all travel health inquiries were collated and analysed. One hundred and forty-four individual recommendations were made from 75 inquiries covering 44 countries, at an average of 3.2 individual recommendations each working day. The findings are presented below with the aim of assessing the impact and role of the travel inquiries on the functioning of the PHU. This report also highlights some of the problems encountered when providing advice to potential travellers.

\section{METHODS}

A travel health inquiry form (Form A) was designed by the PHU and used for all inquiries. The form required the name and address of the client, date of inquiry, date of birth, month of departure, countries to be visited and length of stay in those countries. All travel inquiries were received by phone and immediately directed to a relevant staff member. At no time was the information given over the phone anything more than a general recommendation, which needed to be discussed and tailored to the individual's needs by his/her medical practitioner. This was emphasised to every client, who was then mailed the completed form $\mathrm{A}$ and a copy of the Commonwealth Department of Community Services and Health booklet Fit To Travel And Return.

The travel inquiries were handled in three ways:

i) Standard inquiries - relevant recommended immunisation guidelines for travellers were taken directly from the Commonwealth Serum Laboratory's (CSL) 1989 Country by Country Guide to International Travel Immunisation. From December, the second edition of the guide was used. These inquiries were dealt with mainly by the Public Health Nurse.

ii) Complex inquiries - these often consisted of multiple African or South American countries, and reference would be made to MASTA - a computerised database of medical information for travellers. The client would be phoned back by the PHU nurse with information which was more detailed but still within the form A format.

iii) Doctor inquiries - these were dealt with by the medical staff and MASTA was used as necessary. Form A plus the relevant MASTA information was sent to the doctor.

\section{RESULTS}

There were 75 individual inquiries comprising 144 destination countries and encompassing 44 different countries. Fifty-seven per cent of the clients were males and 43 per cent females. Average age of the clients was 29 years for males and 32 years for females, with a range from less than 1 year to 73 years. There was an uneven distribution of age and gender, with more males than females in all age groups except for the 30-39 age group.

The mean duration of travel was 10 weeks. Most frequent length of stay overseas was three weeks (17.4 per cent), followed closely by four weeks and nine weeks (14.6 per cent and 13.2 per cent respectively).

Hornsby local government area had the most inquiries (15.4 per cent), followed by Blacktown (14.6 per cent) and Parramatta (11.9 per cent). Analysis of the point of inquiry revealed that 61.6 per cent came from residents within the area serviced by the Western Sector PHU, while 16.8 per cent and 14.7 per cent came from the bordering areas of Northern and South Western Area Health Services respectively.

Thailand was the most popular country, accounting for 11.1 per cent of inquiries, followed by India (6.9), and then Brazil, Argentina, Singapore and Malaysia (4.9). South-East Asia was the largest geographical area, with 34.7 per cent of country inquiries to that area.

There was almost total uniformity in the recommendations about the following vaccines: diphtheria, polio, tetanus, yellow fever and malaria prophylaxis. There was poor uniformity in the recommendations for rabies, hepatitis A, hepatitis B, typhoid, cholera, Japanese encephalitis and meningococcal meningitis.

\section{DIsCUsSION}

A number of problems were identified on analysis of the data - principally the difficulty in giving relevant and consistent travel health recommendations over the phone.

The general recommendations we purported to give as they were written in the CSL guide were exactly what the name states: recommendations only, but the import of this was and is very difficult to convey to the travelling public. This was particularly the case with certain vaccine-preventable diseases such as rabies, cholera, meningococcal meningitis, hepatitis A and Japanese encephalitis, where the vaccines were often recommended although this was not always the most appropriate advice.

The inherent limitations of the service were best illustrated by the recommendations to travellers entering countries with endemic rabies. The rabies vaccine was recommended according to the protocol while what was really required was not a vaccination but education about the possible risk of rabies to the individual traveller. Travel health education must cover both precautions and the follow-up emergency actions and seeking of medical advice.

Preventive education for the traveller is also needed to minimise the risk of infection with hepatitis A, cholera, typhoid and other food-borne illnesses. This would include advice on handwashing, water quality and food selection and cooking. In many instances, effective education would obviate the need for vaccines. 


\section{Travel vaccination headaches}

\section{Continued from page 129}

The seasonal variation of diseases and the increasing number of mainstream travellers going to remote and often disease-infested areas has led to special problems with giving advice about the meningococcal and Japanese encephalitis vaccines. CSL recommendations for the two diseases present problems as they do not take into account the type and duration of travel, standard of accommodation, individual itineraries and activities of the traveller. The latter needs to be addressed in a travel health consultation where the balance between the risk of infection and the efficacy, safety and cost of the vaccine can be judged effectively. Japanese encephalitis vaccine is further complicated because the Commonwealth Department of Community Services and Health in Canberra has to approve its use.

It is difficult to define the areas where meningococcal meningitis is present in epidemic proportions and thus provide a comprehensive service. The latter could be addressed by performing weekly updates on all the country inquiries but it would be both too expensive and difficult to implement as the information would require a medical interpretation dependent on a number of factors in the traveller's trip. This cannot be delivered over the telephone. Instead, the information was designed to inform the member of the public and give standard recommendations which could be used as a guide in consultation with the local doctor.

The dramatic rise in the number of people travelling overseas has resulted in an increase in the numbers receiving vaccines and thus the number of adverse sideeffects, which can range from a mild flu-like illness to the more severe Steven Johnson syndrome. It is therefore important that only travellers 'at risk' receive appropriate vaccines. Specialised travel clinics and general practice training are needed so uniform advice is given and adverse reactions can be monitored and acted on.

Recommendations for malaria prophylaxis resulted in a number of problems: with standard inquiries, travellers often requested specific information on drugs and dosages. This was most probably because general practitioners were unsure of which antimalarial drugs to prescribe, as was confirmed by the large number who contacted us about appropriate drugs. It was found that few had a copy of the National Health \& Medical Research Council's Malaria Guidelines for General Practitioners. Recommendations for antimalarial prophylaxis were also complicated by the MASTA reference not being as comprehensive and up-to-date as had been expected.

The major difficulty with yellow fever vaccine was the poor publicity about its availability. Neither members of the public, travel agents nor local doctors knew where to get it. This reflects the lack of information travellers are given by their travel agencies about overseas health risks and vaccine requirements. Travellers are unaware of the time, cost and need for vaccinations and antimalarial tablets so there is no planning of the latter to ensure the optimal time interval is available in the delivery of the vaccinations.

Travel vaccination record books are used by some of the travel medicine practices but travellers, travel agents and general practitioners are confused about the need to have one and have it stamped for overseas travel. This strengthens the argument for some consultation with the travel industry and the College of General Practitioners over the development of a basic overseas travel kit aimed at alleviating these problems.

The last area for discussion is how the existing PHU travel information services are going to develop and change. We need policy guidelines on what type of common service all the PHUs will be required to provide. This would identify the target population and the detail of information required and give an indication of what level of resources the PHUs should expend on the service. It is important that all PHUs be required to provide a specified minimal service so one unit does not inherit all the inquiries and so a critical level of expertise in travel health develops. It is interesting to note that the Hornsby local government area in the Northern Sydney Area Health Service was where most of our inquiries originated.

\section{CONCLUSION}

A service answering travel health inquiries is both time-consuming and limited in the information it can provide. General recommendations can be given over the telephone, followed by a letter confirming the details but this cannot replace a travel health consultation where the traveller should be given both the required immunisations and - more importantly - be educated about the possible health risks of the overseas travel. The latter is not happening and this means people ring the PHU to get information which cannot safely and effectively be delivered over the phone.

Issues that must be addressed are:

- the level of service to be provided to the public and local GPs

- the need to define what a general practitioner travel health consultation should entail

- what information they and the travel agencies should provide to minimise adverse health outcomes to the public.

\section{Elizabeth Sullivan}

Formerly Medical Officer of Health

Western Sector Public Health Unit

\section{PUBLIC HEALTH BULLETIN EDITORIAL STAFF}

The Bulletin's editorial advisory panel is as follows:

Dr Sue Morey, Chief Health Officer, NSW Health Department; Professor Stephen Leeder, Professor of Community Medicine, University of Sydney; Professor Geoffrey Berry, Professor of Epidemiology \& Biostatistics, University of Sydney; Dr Christine Bennett, Associate Director, Services Planning, Service \& Capital Planning Branch, NSW Health Department; Dr Michael Frommer, Epidemiologist, Epidemiology \& Health Services Evaluation Branch; Jane Hall, Director, NSW Centre for Health Economics, Research and Evaluation, Department of Community Medicine, Westmead Hospital; and Michael Ward, Manager, Health Promotion Unit, NSW Health Department.

The editor is Dr George Rubin, Director, Epidemiology \& Health Services Evaluation Branch, NSW Health Department. Please send your articles, news, comments or letters to him at Locked Bag 961, North Sydney NSW 2059 or fax (02) 3919232 . Suggestions for improving the content of the Bulletin are welcome.

Design - Health Public Affairs Unit, NSW Health Department. 